\title{
Cerebral Atrophy and Convulsive Seizures after Recovery from Cerebral Edema and Coma in a Patient with Fulminant Hepatitis B
}

\author{
Suguru HondA*, Akira TANIGUCHI, Katsumasa MuraI and Shigeki KuzUHARA
}

\begin{abstract}
We report a 48-year-old woman who developed convulsive seizures and cerebral atrophy after recovery from fulminant hepatitis B with coma and cerebral edema at the acute stage. Neurological disturbances and cerebral signs are rare sequelae of fulminant hepatic failure (FHF); only a few cases have reported in the literature. Cortical laminar necrosis secondary to cerebral edema or delayed neuronal death due to toxic substances may have caused delayed brain atrophy and epileptogenesis.

(Internal Medicine 40: 255-258, 2001)
\end{abstract}

Key words: fulminant hepatic failure, neurological complication, brain computed tomography, electroencephalography

\section{Introduction}

Viral hepatitis and drugs are major causes of fulminant hepatic failure (FHF). FHF occurs in less than $1 \%$ of patients with viral hepatitis, and the mortality rate is extremely high. However, the diseased liver is potentially reversible, and permanent neurological disturbances in those who survived FHF occur rarely. To our knowledge, there are only a few reported cases of FHF with cerebral edema and coma at acute stage and subsequent cerebral atrophy and neurological complications after recovery. We report a survivor of FHF due to hepatitis B with cerebral edema who later developed cerebral atrophy and convulsive seizures.

\section{Case Report}

A 48-year-old woman was admitted for jaundice and coma on August 25, 1992. She had been healthy until the admission and did not have past history of alcoholism, blood transfusion, or drugusage. Her blood pressure was $164 / 104 \mathrm{mmHg}$, pulse rate $120 \mathrm{bpm}$, and temperature $37.7^{\circ} \mathrm{C}$. She was comatose without spontaneous motor activity and speech, and withdrew the extremities with deep painful stimuli (Glasgow Coma Scale score; eye opening 1 , motor response 3 , verbal response 1 ). Her pupils were isocoric and reacted sluggishly to light. Deep tendon reflexes of the lower extremities were hyperactive, and the Babinski sign was positive bilaterally.

Laboratory data on admission indicated severe hepatic failure as follows; AST 2,549 IU/l, ALT 6,310 IU/l, total bilirubin $9.5 \mathrm{mg} / \mathrm{dl}$, blood sugar $87 \mathrm{mg} / \mathrm{dl}$, and ammonia $200 \mu \mathrm{mol} / l$ (normal 3-40 $\mu \mathrm{mol} / l$ ). IgM HBc antibody was positive. Brain computed tomography (CT) on admission showed findings indicative of severe diffuse cerebral edema, including loss of cortical sulci, low density of the white matter, and narrowing of the lateral and third cerebral ventricles (Fig. 1A). Electroencephalography (EEG) showed periodic synchronous discharges (PSD) on a low voltage background activity in all leads (Fig. 2).

Though the cause of infection was not clarified, a tentative diagnosis of FHF due to hepatitis B was made, and an intensive therapy with intravenous glucagon and insulin in combination with plasma exchange transfusion was started. Convulsive jerks occurred in the extremities and face several times a day, and they were controlled with repeated intravenous injections of $5 \mathrm{mg}$ diazepam. On the 2 nd hospital day, the liver function improved markedly; AST $234 \mathrm{IU} / l$, ALT $874 \mathrm{IU} / l$, total bilirubin $5.2 \mathrm{mg} / \mathrm{dl}$, blood sugar $256 \mathrm{mg} / \mathrm{dl}$, ammonia $100 \mu \mathrm{mol} /$ $l$. Massive fresh-frozen plasma (FFP) was administered since cutaneous purpura had appeared. Her condition and laboratory data improved gradually. She finally recovered from FHF on the 20th day, and the serum ammonia level decreased to 44 $\mu \mathrm{mol} / l$.

She had been well until the 32nd day when she suddenly had a generalized convulsive seizure with loss of consciousness which lasted for 20 minutes. Brain CT revealed marked

From the Department of Neurology, Mie University School of Medicine, Tsu, *present address; Department of Neurology, Matsusaka Saiseikai Hospital, 1-156 Asahimachi, Matsusaka, Mie 515-8557

Received for publication September 8, 1999; Accepted for publication April 23, 2000

Reprint requests should be addressed to Dr. Suguru Honda, the Department of Neurology, Matsusaka Saiseikai Hospital, 1-15-6 Asahimachi, Matsusaka, Mie 515-8557 

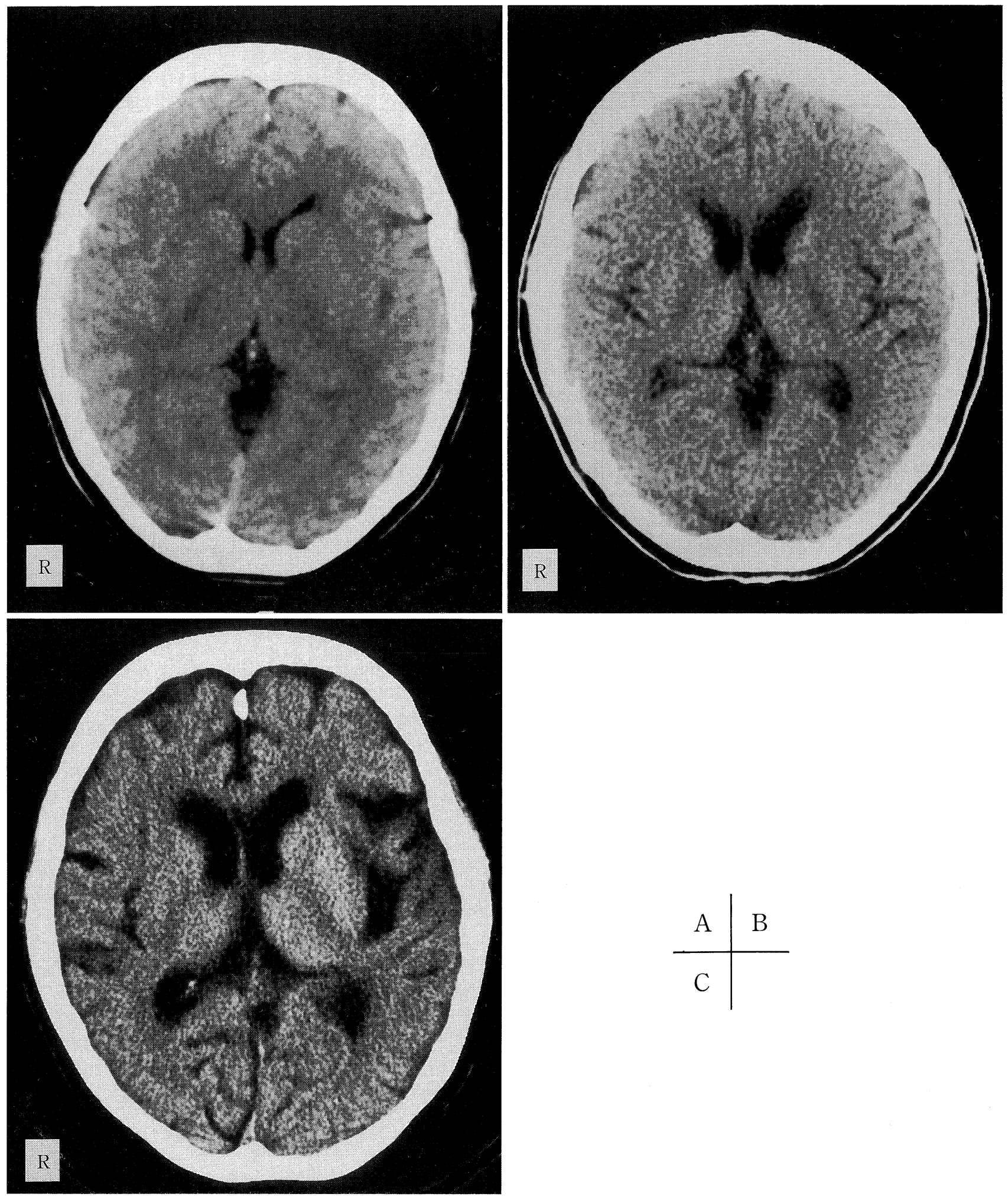

Figure 1. Follow up Brain CTs. (A) At the acute stage (August 28, 1992), there is marked cerebral edema with low density of white matter, narrowing of the lateral ventricles, and disappearance of the cortical sulci. (B) At the recovery from the coma (September 30, 1992), cerebral edema is markedly improved. (C) Marked cerebral atrophy is evident 5 years after the onset (December 24, 1997). 




Figure 2. EEG during coma shows high voltage synchronous bursts on the low voltage background activities.

improvement of cerebral edema (Fig. 1B). Brain magnetic resonance imaging (MRI) on October 16, 1992 showed no findings indicative of laminar necrosis, borderzone infarction, or degeneration of globus pallidus.

A daily dose of $1,200 \mathrm{mg}$ valproic acid and $1,000 \mathrm{mg}$ primidone relieved the convulsive seizures. On December 17, 1997, she was readmitted for poor control of convulsive seizures. Neurological examination revealed hyperactive deep tendon reflexes and a positive Babinski sign on the left side. She complained of difficulties in mental concentration, and her short time memory was slightly disturbed. Verbal intelligence quotient (VIQ) was 67, and performance intelligence quotient (PIQ) 51 , on the Wechsler Adult Intelligence Scale-revised (WAISR). Calculation and digit retention were markedly disturbed. Laboratory data including liver functions, serum ammonia level, blood gas, cerebrospinal fluid were within normal limit, and serum levels of valproic acid and primidone were within therapeutic concentrations.

Brain CT (Fig. 1C) as well as brain MRI demonstrated moderate cerebral cortical atrophy with enlargement of the third and lateral ventricles. An interictal single photon emission com- puted tomography (SPECT) with ${ }^{123}$ I-IMP revealed a marked decrease in the blood flow of the left temporo-occipital lobe. EEG showed basic activities of $9 \mathrm{~Hz} \alpha$ waves without epileptic discharges. A daily oral dose of $10 \mathrm{mg}$ diazepam markedly reduced convulsive seizures.

\section{Discussion}

Cerebral edema, one of the major complications of FHF (14) causes death in 60 to $70 \%$ of FHF patients (5-7). Neuropsychiatric disturbances of survivors are rare since FHF and cerebral edema are usually reversible. Fiasse et al (8) reported a patient who survived FHF by exchange transfusion, and who had developed aphasia and acalculia. Tubbs et al (9) reported persistent psychiatric disturbances, and atrophy of the cerebral cortex and optic nerve on brain CT in a man who had survived FHF of unknown etiology. Toda et al (10) reported a survivor of FHF after blood transfusion, who had developed epileptic attacks, character changes, mental deterioration, and generalized brain atrophy on brain CT. In the present case, brain CT revealed marked swelling of the brain indicative of severe cerebral edema during coma at the acute stage, and marked cerebral atrophy five years after recovery.

Pathogenesis of cerebral edema in FHF remains unclarified. As to the production of cerebral edema, two hypotheses have been proposed. One is vasogenic mechanism which refers to a direct leakage of water into the brain tissue by disruption of the blood-brain barrier, and the other is cytotoxic mechanism which refers to an increase in water uptake into the brain tissue by cellular alterations $(3,11)$.

The mechanism of cerebral atrophy secondary to cerebral edema, and epileptogenesis in FHF is probably multifactorial. At the acute stage of FHF, she had no episodes of respiratory failure or shock which could cause hypoxia and subsequent cerebral atrophy. At the chronic stage when convulsive seisures occurred, hypoxemia and hyperammonemia were absent. Either toxic substances during hepatic failure or ischemia secondary to cerebral edema, or both can damage the brain tissue, and result in cerebral atrophy at the chronic stage. According to Kato et al (12), brain swelling in FHF is usually caused by cytotoxic edema, and hypoxia and hemorrhages secondary to brain swelling may also be noted. In the present case, the brain MRI at the acute stage showed no findings indicative of laminar necrosis, borderzone infarction, or degeneration of globus pallidus. However, low voltage slow background activities and PSD on EEG indicated diffuse severe damage of the cerebral cortex. Despite the absence of neuroimaging evidence, either cortical laminar necrosis or delayed neuronal death remains a possible cause of brain atrophy and epileptogenesis at the chronic stage.

More attention must be paid to the neurological complications of the survivors of FHF since with the recent development of new therapies for FHF more and more FHF patients have been cured. 


\section{HoNDA et al}

Aknowledgements: We thank Dr. Katsuya Shiraki, Dr. Takashi Tanigawa, and Prof. Dr.Takeshi Nakano (First Deparetment of Internal Medicine, Mie University, Japan) for their help and advice in preparing the manuscript.

\section{References}

1) Wijdickes EF, Plevak DJ, Rakela J, Wiesner RH. Clinical and radiologic features of cerebral edema in fulminant hepatic failure. Mayo Clin Proc 70: 119-124, 1995 (see comments).

2) Rakela J, Lange SM, Ludwig J, Baldus WP. Fulminiant hepatitis: Mayo Clinic experience with 34 cases. Mayo Clin Proc 60: 289-292, 1985.

3) Hoofnagle JH, Carithers RL Jr, Shapiro C, Ascher N. Fulminant hepatic failure: summary of a workshop. Hepatology 21: 240-252, 1995.

4) Watanabe A, Shiota T, Tsujii T. Cerebral edema during hepatic encephalopathy in fulminant hepatic failure. J Med 23: 29-38, 1992.

5) Gazzard BG, Portmann B, Murray-Lyon IM, Williams R. Causes of death in fulminant hepatic failure and relationship to quantitative histological assessment of parenchymal damage. Q J Med 44: 615-626, 1975.

6) Ede RJ, Gimson AE, Bihari D, Williams R. Controlled hyperventilation in the prevention of cerebral oedema in fulminant hepatic failure. $J$ Hepatol 2: 43-51, 1986 .

7) Forbes A, Alexander GJ, O'Grady JG, et al. Thiopental infusion in the treatment of intracranial hypertension complicating fulminant hepatic failure. Hepatology 10: 306-310, 1989.

8) Fiasse R, Collignon R, Bietlot A, et al. Treatment of fulminant hepatitis with coma by exchange transfusion. Study of 9 patients, including 1 with neurological sequelae in the cortical functions (author's transl). Acta Gastroenterol Belg 37: 12-39, 1974.

9) Tubbs H, Parkes JD, Murray-Lyon IM, Williams R. Cortical and optic atrophy following fulminant hepatic failure. Med Chir Dig 6: 75-77, 1977.

10) Toda $C$, Chiba $T$, Matsuda $Y$, Inatome $T$, Inoh $T$, Fujita $T$. A case of brain atrophy after fulminant hepatic failure. Am J Gastroenterol 78: 446-448, 1983.

11) Blei AT. Pathogenesis of brain edema in fulminant hepatic failure. Prog Liver Dis 13: 311-330, 1995.

12) Kato M, Hughes RD, Keays RT, Williams R. Electron microscopic study of brain capillaries in cerebral edema from fulminant hepatic failure. Hepatology 15: 1060-1066, 1992. 